\title{
Influence of operating parameters on sewage sludge drying in a paddle dryer: design of experiments for the determination of hold- up and water content profiles
}

\author{
M. Milhé*, M. Sauceau, P. Arlabosse \\ Université de Toulouse; Mines Albi; CNRS; Centre RAPSODEE \\ Campus Jarlard, F-81013 Albi, France \\ Email of the corresponding author: mmilhe@mines-albi.fr
}

\begin{abstract}
In previous works, solids hold-up proved to be the most influential parameter of a stochastic flow model of a continuous paddle dryer: we thus aimed at correlating it with operating parameters. A fractional factorial Design of Experiments (DoE) was implemented, in which dryer slope, stirrer speed, overflow level and sludge flow rate were initially selected as factors. The DoE was then completed after reducing the number of influent parameters and their interactions, using surface response methodology (RSM), to get a predictive relationship for solids hold-up determination. The nonlinear influence of overflow height and dryer slope was confirmed by the different water content profiles obtained during the experiments. Finally, the key role of dry solids residence time on final sludge water content was highlighted, with an almost linear relationship. These results emphasize the importance of hold-up for modeling purposes: this parameter has an effect on sludge flow but also on the contact area between sludge and dryer walls.
\end{abstract}

Keywords: Paddle dryer; Design of experiments; Solids hold-up; Empirical correlation; Water content profile 


\section{Introduction}

Sewage sludge (SS) management is a matter of growing importance: SS production keeps increasing with population growth and legislation restrictions. Storage and hygiene issues, as well as landfilling limitations, contribute to making drying a necessary step in SS treatment and valorization [1][2]. However, SS is a product quite difficult to handle, as it undergoes a transition to a sticky state during its drying [3]: this is potentially damageable for industrial dryers where moving equipment is involved, such as contact dryers. For this reason, paddle dryers are recognized as well adapted tool for SS contact drying: thanks to the wedge-shaped paddles, high shearing of the product is realized and in situ clogging is avoided [4]. Moreover, this kind of indirect dryer offers the advantages of compactness and low exhaust volumes. In spite of the important number of installations functioning at an industrial scale, design and operation of paddle dryers mostly rely on manufacturers' know-how [5]. Different ways of energetic valorization for SS are under study or actually in use, requiring various minimal water content in sludge [6]. In these processes, drying is very energy-consuming, with around $900 \mathrm{kWh} /$ ton of water removed. Limiting the extent of drying to the minimum needed, instead of the values of more than $90 \%$ of solid content often reported in industrial installations, could lead to significant energy savings and improvements of the viability of new pathways for energy recovery [7]. In this framework, a comprehensive modeling of the drying process in a paddle dryer would constitute a very interesting tool in order to identify the critical parameters and their influence on dryers' performances. 
Few studies focused on the description of sludge drying in paddle dryers in continuous operation: while Arlabosse et al. described drying kinetics along an industrial scale installation with a simple empirical model assuming plug flow of sludge [5], Tazaki et al. highlighted the importance of back-mixing in such installations via a Residence Time Distribution (RTD) study [8]. More recently, Charlou et al. developed a methodology for measuring experimental RTD with a good repeatability in a lab-scale pilot installation [9]. These experimental RTDs were then compared to the ones obtained by a Markov chains modeling of the reactor. In this model, each paddle constitutes a continuous perfectly stirred tank reactor, exchanging forward and backward sludge flow with its direct neighbors, as in Tazaki's work, but sludge flow is considered as a stochastic process [10]. Very good agreements were observed between simulated and experimental RTDs, after optimization of the two main model parameters. These parameters are the dry solids hold-up $H u$ in each cell and the recirculation coefficient $R$ between the cells: both were considered constant along the dryer, i.e. for all the cells. If this is the only way for obtaining a value of the recirculation coefficient $R, H u$ however can be experimentally measured. The comparison of these data with the values obtained after optimization of the model showed that this optimization was not necessary as far as $H u$ is concerned. Moreover, optimized $R$ values lie between 2 and 5 in the range of operating conditions tested.

A numerical parametric analysis of this Markov flow modeling highlighted the great influence of $H u$ on sludge flow pattern, while $R$ only has a limited influence [10]. In order to obtain a model of sludge drying in a paddle dryer with no adjustable parameters, it is thus very important to be able to estimate $H u$ for a given set of operating parameters. A first step is to determine which parameters actually have an influence on $H u$ value; then, an empirical correlation based on experimental results could be deduced. This can be achieved with a parametric study, by applying 
perturbations to a single operating parameter at a time, starting from a reference experiment. However, this systematic approach is only applicable when the number of parameters and the range of operating conditions are limited. When one has to work with a limited number of experiments, setting up a Design of Experiments (DoE) is more advisable. This term encompasses an ensemble of methodological tools allowing an experimenter estimating any parameter influence on a given output of an experiment. Moreover, by carefully choosing parameters values, DoE give access to statistical analysis of the results, giving more insight into their significance [11-13].

In this paper, we studied the influence of several operating parameters on drying performances of municipal sewage sludge in a lab-scale continuous paddle dryer. We first implement a design of experiments, leading to an empirical correlation for estimating dry solids hold-up for a given set of operating conditions. Then, the influence of this hold-up on experimental water content profiles and final water content of sludge is studied.

\section{Material and methods}

\section{Sludge characteristics}

The sludge used in this study comes from the Albi city WWTP (60 000 population equivalent). The effluent is treated via a biological-chemical phosphorus and nitrogen removal process. The activated sludge is flocculated with a cationic polymer, thickened and anaerobically digested for three weeks. The digested sludge is then flocculated and mechanically dewatered in centrifuges, which allow reaching water contents between 3.5 and $4 \mathrm{~kg} / \mathrm{kg}$. The results presented in this study 
were obtained with sludge sampled over a period of three months during which no change in the operating conditions of the WWTP was reported. Sludge was stored in air-tight containers in a cold room at $+4^{\circ} \mathrm{C}$ for at least two days before each experiment for stabilization of the biological activity [14], and was not kept for more than two weeks.

\section{Paddle dryer description}

Experiments were conducted on a continuous pilot-scale paddle dryer (Figure 1): it is composed of a 1 meter long U-shaped jacketed trough housing a shaft equipped with 18 regularly spaced wedge-shaped paddles; scrapers are situated between the paddles in order to increase mixing and avoid crust formation on their surface. Both the jacket and the shaft are electrically heated in three separate zones, with regulation thermocouples placed near the stainless steel surface. Three traps situated on top of the trough allow sampling of drying sludge during or after the experiments. These traps as well as the trough are insulated with $5-\mathrm{cm}$ thick rock wool to avoid heat losses. Sludge and superheated steam, used a sweeping gas, are injected from one end of the trough, above the paddles. Exhaust gases are entrained from the other end of the pilot through an activated carbon filter before being released outside. Sludge is fed from a 401 hopper by means of a Moineau pump which flow rate $Q$ can be accurately controlled. Dried sludge is discharged above an overflow which height $H$ can be varied from the shaft axis height to the top of the paddles. The whole installation slope $\theta$ can be varied between 0 and $+5^{\circ}$ and rotational speed of the shaft $N$ can also be varied between 10 and $60 \mathrm{rpm}$.

\section{Experimental procedure}

5 
The installation is equipped with different sensors allowing recording temperatures and electrical power in three different zones along the dryer, torque applied by the motor as well as mass of dried sludge at the outlet. This monitoring can help knowing whether a steady state operation has been reached. Indeed, reaching a steady state during SS drying experiments is not straightforward: one cannot only rely on sludge outlet flow, since its water content can vary during transitory periods. Moreover, water content measurement requires several hours at least (24h at $105^{\circ} \mathrm{C}$ according to the norm NF EN 15934). We could overcome this issue by monitoring continuously the torque applied by the stirrer along with the mass at the outlet. Figure 2 illustrates the relationship between torque and sludge flow rate at the outlet of the dryer (averaged on 30 min periods). The first part of the graph illustrates a period of stability of both torque and sludge outlet flow rate: thanks to RTD experiments, we have observed that this behavior corresponds to a steady-state regime in the dryer [15]. After $4 \mathrm{~h}$ of operation, an increase in torque value is accompanied by a decrease in average flow rate and conversely. This indicates that an accumulation of sludge occurred, followed by a partial purge of the material in the reactor. Such behavior is uneasy to anticipate and to explain, but is probably linked to the stickiness of the sludge during drying [3]. In order to ensure that the remaining sludge in the dryer corresponds to that of a steady-state operation, we waited for the torque and outlet flow rate to be stable for at least $2 \mathrm{~h}$. Once a steady-state operation was achieved, sludge feed was stopped and the outlet was closed in order to isolate the material in the dryer. Sludge was then sampled at 9 regularly spaced spots along the dryer in order to obtain a water content profile during steadystate operation. Stirring and heating were maintained for $2 \mathrm{~h}$ in order to avoid the formation of sticky lumps inside the dryer. Dry sludge was eventually recovered and fully dried in an oven for $24 \mathrm{~h}$ at $105{ }^{\circ} \mathrm{C}$ for the dry solids hold-up $H u$ determination. 


\section{Design of Experiments}

In a DoE, a well-chosen set of experiments allows one studying the effect of $n$ parameters $X_{i}(i=$ $1, \ldots, n)$ on a measured response $Y$. The statistical analysis of the results leads to expressing this response as function of the parameters (Eq. (1)).

$Y=f\left(X_{1}, X_{2}, \ldots, X_{n}\right)$

Different kinds of DoE exist and their usage depends on the problem studied and its specific experimental limitations. If no constraints exist, an experimenter would start with a factorial design (FD), where each parameter will take two values. These values define the boundaries of the experimental domain. An exhaustive study of the influences of the parameters and their interactions on the response would then imply to realize $2^{n}$ experiments, which would allow establishing an empirical correlation as in Eq. (2). This is the approach realized with a full FD, in which all interactions effects can be evaluated, with potential interactions between up to $n$ parameters. If 4 parameters are considered, this implies carrying out 16 experiments, since $Y$ would be estimated as a multi-linear expression of 16 factors.

$Y=K+\sum_{i=1}^{n} \alpha_{i} X_{i}+\sum_{i=2}^{n} \sum_{j}^{i-1} \alpha_{i, j} X_{i} X_{j}+\sum_{i=3}^{n} \sum_{j=2}^{i-1} \sum_{k}^{j-1} \alpha_{i, j, k} X_{i} X_{j} X_{k}+\cdots$

\section{Fractional factorial design}

If one needs to decrease the number of experiments, it is possible to carry out a fractional FD, generally identified by the notation $2^{n-k}$. In a fractional FD, the number of experiments is divided 
$2^{k}$-fold. However, the estimation of the influences of the factors is more subtle. For example, it is possible to realize a $2^{4-1}$ fractional FD, which gives access to a relation in the form of Eq. (3).

$Y=\sum_{i=1}^{8} \alpha_{i} A_{i}$

In this relationship, 8 factors $\left(A_{i}\right)$ are considered for the description of $Y$ instead of 16 . These factors actually stand for the combined influences of two factors or interactions: they are aliased. In practice, this means that between each experiment two parameters are modified and that the change observed in the response is a combination of the influence of these parameters or their interactions. Depending on the matrix of experiments set up, it is possible to choose the different aliases so as to combine factors which have a priori a negligible influence with those that are likely to be very influent. $3^{\text {rd }}$ and higher order parameters interactions (i.e. interactions between 3 or more parameters) are often neglected: it is then advisable to alias the constant term and main factors with these high order interactions. More information on aliases theory can be found in literature [11]. This approach can be used in a first step in order to rule out factors which have little or no influence on the response before setting up a more complex DoE.

In this study, we started from a fractional FD with 4 operating parameters, i.e. $H, Q, N$ and $\theta$. The objective of this DoE is to investigate whether or not these parameters as well as their interactions have an influence on $\mathrm{Hu}$. These parameters are not correlated, and the response can be measured accurately. Apart from $H$ and $\theta$, which influence on solids hold-up was expected, our objective was to verify whether $N$ and $Q$ or their interaction was influencing the solids holdup as it is the case in rotary drums for example [16].

Wall temperature was not considered in this study because its effect on dry solids hold-up would have been very difficult to rely to experimental observations. Indeed, for lower drying 8 
temperatures sludge drying rate would be lower; if the other parameters would be kept constant, the dryer would be filled with sludge having average water content higher than for a higher wall temperature. Thus, the dry solids hold-up could be lower for the same volumetric filling of the dryer. However, including this fifth parameter would have made the DoE more difficult analyze since the number of experiments would have stayed the same; the corresponding fractional FD would have been $2^{5-2}$. We then chose to work under a constant wall temperature, in order to have a clearer interpretation of operating parameters influences on the hold-up, which is the key parameter for dryer performance for a given drying temperature. The temperature of $160^{\circ} \mathrm{C}$ used in the experiments corresponds to a classical temperature for industrial applications where steam is employed as a heating medium. We expect the results of this study to be the same in a qualitative point of view for different wall temperatures. Sweeping gas flow rate was not taken into account since steam is only used as a sweeping gas and not as a heat transfer medium; indeed, it was concluded from other experiments that steam temperature had no impact on the water content profile along the dryer; hance, the convective heat transfer is not significant in this configuration. Finally, the origin and initial water content of the sludge are not in the frame of this study.

The experimental procedure described previously is very time-consuming, because of long periods for heat up and cooling down of the installation, and because of slow evolutions towards a steady-state operation. For that reason, and with the four parameters identified, we chose to work according to a fractional FD $2^{4-1}$, thus limiting the number of experiments to 8 . This enabled us to work with a single batch of sludge, avoiding possible bias linked to aging of sludge or to a possible variation in sludge properties at the outlet of the WWTP. 


\section{Circumscribed central composite design}

Composite designs are a category of response surface methodology designs that can complete an initial FD. One can establish a correlation of $2^{\text {nd }}$ order as in Eq. (4) by adding experiments to the initial experimental domain. This is interesting for problems of optimization or when the evolution of the response is not likely to be linear with respect to the different parameters involved. In such designs, $3^{\text {rd }}$ and higher order interactions are generally not taken into account [13].

$Y=K+\sum_{i=1}^{n} \alpha_{i} X_{i}+\sum_{i=2}^{n} \sum_{j}^{i-1} \alpha_{i, j} X_{i} X_{j}+\sum_{i=1}^{n} \alpha_{i}^{\prime} X_{i}^{2}$

Here again, several design types can be set up, that will influence some statistical properties of the empirical correlation obtained. In this study, we worked according to a circumscribed central composite design (CCCD). In a CCCD, parameters take additional values outside the initial experimental domain, thus enlarging it. For that reason, care must be taken when designing the initial experimental domain of the FD if the objective is then to implement it into a CCCD. The additional points are situated at a distance $\alpha=\sqrt[4]{2^{n}}$ from the center of the experimental domain; this allows obtaining a constant error on the experimental domain studied. Moreover, carrying out multiple central experiments allows a better estimation of the variance of the regression, i.e. the error of the prediction. The experimental domain of a CCCD is schematically represented in Figure 3 for two parameters.

\section{Results and discussion}

\section{Fractional factorial design with 4 parameters}


The DoE operating conditions are gathered in Table 1 in absolute and coded values, along with the corresponding measured solids hold-up. The coded values are calculated as in Eq. (5), where $X_{0}$ is the value of a parameter $X$ at the center of the experimental domain, $X^{\prime}$ is its coded value, and $\Delta X$ corresponds to the limit of the experimental domain. All the results presented in this paper are expressed in a non-dimensional form, with coded values $X^{\prime}$ for the parameters and the corresponding coefficients $\alpha_{i}$.

$X^{\prime}=\frac{X-X_{0}}{\Delta X}$

The experimental domain defined here is relatively narrow. However, parameters values were chosen in order to be able to carry out all the potential experiments of the subsequent CCCD, where the coded values would be laying between $\alpha=1.4$ and $\alpha=2$ depending on the number of retained parameters.

For each experiment, the response can be expressed as in Eq. (3). Solving this set of equations gives access to the 8 coefficients corresponding to 8 variables describing the response. As previously stated, in a fractional FD parameters and their interactions influences are aliased. In our case, the coefficients obtained and the corresponding aliases implied by this DoE are gathered in Table 2. We neglected $3^{\text {rd }}$ and $4^{\text {th }}$ order interactions (i.e. between 3 and 4 parameters respectively), which simplifies the interpretation of the DoE results. In the following, we thus consider that the first five coefficients $\left(\alpha_{1}\right.$ to $\left.\alpha_{5}\right)$ are only related to the constant term and the main parameters, while the last coefficients $\left(\alpha_{6}\right.$ to $\left.\alpha_{8}\right)$ stand for 2 aliased interactions each.

Several DoE software can give access to statistical treatment of these results: in this study we worked with Nemrodw ${ }^{\odot}$. Figure 4 illustrates the values of the coefficients in Table 2, except for the constant term $\alpha_{1}$ which value is 1637.3. We can see that $H$ and $\theta$ have the greatest 11 
coefficients, i.e. their influence is strongest on the response. Moreover, this influence is statistically significant at the $95 \%$ confidence level, as expressed by the vertical dash-dotted lines. This is easily related to physical effects: an increase in overflow level results in an increase in the volume available for sludge in the dryer which is expressed by the positive value of the coefficient $\alpha_{2}$. On the contrary, an increase in dryer slope will decrease this volume, hence the negative value of the coefficient $\alpha_{3}$.

As illustrated in Figure 4, $N$ and $Q$ influences proved to be largely non-significant. To the authors' best knowledge, there have been no publications concerning this specific aspect of sludge processing in paddle dryers, so this result can only be discussed through analogies with our technologies. Rotary drums appear as the closest configuration, as they are operated for processing divided solids with agitation. In such installations, the mean residence time of solids increases only slightly with the product flow rate $Q$ [16]: this is equivalent to saying that the hold-up increases almost linearly with the product inlet flow rate. This observation is only valid for rotary drums without discharge dam and the equivalent for rotary drums with a discharge dam has not been studied as far as the authors know. The configuration studied here is radically different from the one cited above: whereas in rotary drums, solids flow is mainly driven by product characteristics such as angle of repose and relies on their free flowability [17], this is not the case in SS paddle drying. According to the results of this fractional FD, increasing SS flow rate would not result in accumulating more dry material in the paddle dryer. It is possible that more wet sludge would be present in the dryer, but since at a higher flow rate drying performances are lower, the resulting dry solids hold-up would not be significantly different.

The same qualitative observations can be made regarding the non-influence of the stirring speed $N$ on the dry solids hold-up. Here again, the influence of this parameter on hold-up in rotary 12 
drums without discharge dam is well documented: an increase in the rotation speed will result in a decrease of the hold-up. This is also explained by the increased flowability of the materials in the rotary drum with the rotation speed. On the contrary, in a paddle dryer, the stirrer only has a mixing effect and does not seem to participate in sludge motion. This observation is consistent with what was observed during RTD experiments with different stirring speeds: increasing $N$ from 14 to $28 \mathrm{rpm}$ did not significantly change the RTD pattern while other parameters were kept constant [10].

Concerning interactions terms, a general rule is that a non-influent parameter will not yield significant interactions with other parameters. Here, the only significant interaction possible would be $H^{*} \theta$. The coefficient $\beta_{6}$ can thus be considered as the actual coefficient for this particular interaction (and not for the alias). The non-dimensional coefficient for this interaction term is negative: this means that an increase in either parameter will lower the influence of the other. The influence of slope will thus be low for the greater overflow heights, and conversely the influence of overflow height is lower for the highest slopes while it is important for a slope close to $0^{\circ}$. Based on these results, the problem is simplified by considering only two parameters, $H$ and $\theta$, and their interaction. The hold-up can thus be described by Eq. (6).

$H u=K+\alpha_{H} H+\alpha_{\theta} \theta+\alpha_{H, \theta} H \theta$

The DoE then becomes a full FD with 2 parameters in which each experiment was doubled. The coefficients in Eq. (6) are gathered in Table 3, and the corresponding Pareto chart is illustrated in Figure 5. Coefficient values are the same than previously. However, since there are now more experiments than needed, the system has more degrees of freedom. This reduces the values for the Student test, so p-values calculated for the coefficients in Table 3 are much lower. This 
confirms that both parameters as well as their interaction have a very high probability of being influent at the $95 \%$ confidence level. The standard error on the estimated hold-up is $113.7 \mathrm{~g}$ and the adjusted regression coefficient $R_{a}^{2}$ is 0.985 . The interaction effect between $H$ and $\theta$ is illustrated in Figure 6, which shows that the variation of the hold-up with $H$ is not the same depending on $\theta$ value, and conversely. This correlation appears satisfactory from the statistics

point of view and the coefficients obtained describe well the influence of the operating parameters. However, the value obtained for the constant term was not consistent with previous observations on similar experiments. Moreover, the experimental domain defined in Table 1 is relatively narrow, and it is probable that the influence of $\theta$ is not linear. These issues can be addressed by turning the current full FD into a CCCD.

\section{Circumscribed central composite design with 2 parameters}

This part of the study was realized with a second batch of sludge, all sampled in one time at the same WWTP. Sludge was produced under identical conditions than during the first part of this study. Repeatability experiments were carried out beforehand in order to verify that sludge from these two batches behaved similarly. The objective here is to complete the experiments in order to obtain a correlation of the form of Eq. (7), similar to Eq. (4).

$$
H u=K+\alpha_{H} X_{H}+\alpha_{\theta} X_{\theta}+\alpha_{H, \theta} X_{i} X_{j}+\alpha_{H}^{\prime} X_{H}^{2}+\alpha_{\theta}^{\prime} X_{\theta}^{2}
$$

The CCCD is summed up in Table 4: for the 4 first experiments, the hold-up is the mean of the two experiments corresponding in the first DoE. Indeed, since the influence of $N$ and $Q$ is now neglected, in the initial FD all experiments were actually carried out in duplicates. 4 axis points (experiments 5 to 8 ) were carried out for completing the experimental domain as in Figure 3. 14 
Two central points were finally added (experiments 9 and 10), which show a good repeatability of the experiments. Moreover, hold-up values for these experiments are very different from the value of the constant term issued from the FD, which was almost twice as high. This confirms the need for considering a nonlinear evolution of the hold-up with the operating parameters studied. The coefficients corresponding to Eq. (7) estimated with this DoE are summed up in Table 5. The standard error on the estimated hold-up is $13.7 \mathrm{~g}$, and the adjusted regression coefficient $R_{a}^{2}$ is 0.902. This correlation offers an overall good description of dry solids hold-up, even though the significance of coefficients is a bit lower than the previous one, with p-values comprised between 0.6 and $1.4 \%$ at the $95 \%$ confidence level. The distribution of the error from the correlation in Eq. (7) on all the experiments carried out for this study is illustrated in Figure 7. The error is regularly distributed around 0 , so that the correlation does not appear to be biased; however, it often reaches more than $10 \%$. This low accuracy could result from the assumptions made while carrying out this DoE: for example, while $N$ and $Q$ are considered as non-influent through the statistical analysis, the experiments where these parameters vary do not always end up with repeatable results (see exp. 1 and 2, Table 1). But this also expresses that sludge is a complex biological product, whose rheological behavior is very difficult to understand and anticipate. While great care was taken in obtaining reliable results based on well-controlled experiments, it is possible that some would have shown a poor repeatability.

Contours of the response surface of the correlation in Eq. 7 over the experimental domain of the CCC design are illustrated in Figure 8. $H$ and $\theta$ are expressed in coded values. As expected, the highest $H u$ values are obtained with low slopes and high overflow levels. A limitation to such a simple model also appears in this figure: a minimum for the estimated hold-up appears inside the experimental domain. This is unrealistic because it is not located at the highest value of slope and 
lowest value of overflow height. A practical consequence is that this correlation should not be used for estimating hold-up in this area of the experimental domain.

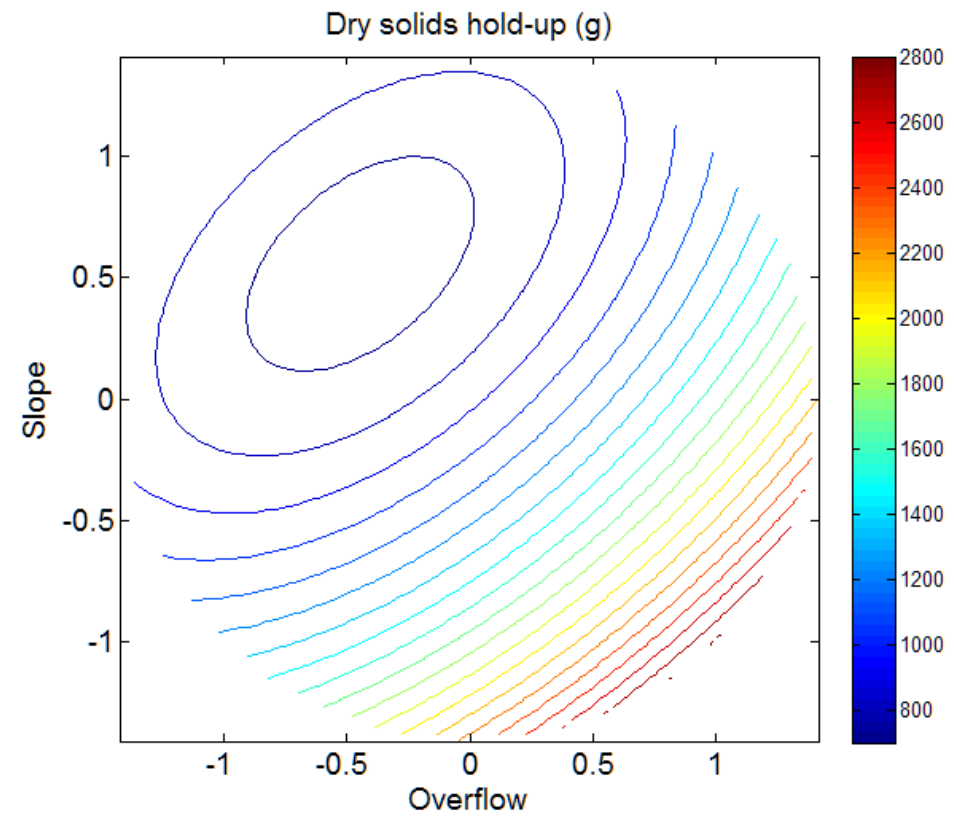

Figure 8: Contours of values for dry solids hold-up estimated from Eq. 7

\section{Influence of operating parameters on water content profiles}

At the end of each experiment, sludge was sampled along the dryer in order to determine the water content profiles. The water content is defined as the ratio of the mass of water to that of dry sludge. The profiles obtained from the additional experiments from the CCCD are plotted for different values of $\theta$ in Figure 9 and $H$ in Figure 10. In some profiles presented the water content decreases at first and seems to increase or stagnate around the middle of the dryer. This is due to the low loading of the dryer in some operating conditions, which implies a poor mixing of the bulk in the middle of the reactor. Granulation occurs at a water content of around $1.5 \mathrm{~kg} / \mathrm{kg}$ : in 
this phase, sludge flows in relatively big lumps and sampling a representative part of the bulk at the corresponding location is not easy.

In Figure 9, the profiles correspond to experiments 7, 8, 9 and 10 (see Table 4), with $\theta$ varying between 1.6 and $4.4^{\circ}$. A first observation is that these profiles show a fairly good repeatability, as the data from experiments 9 and 10 are quite similar. The influence of $\theta$ on water content profiles follows the same trend than that of $H u$ : for 3 and $4.4^{\circ}$ the profiles are similar, while for $1.6^{\circ}$ the water content is much lower particularly after the first third of the dryer. In Figure 10, the profiles correspond to experiments 5, 6 and 9 (see Table 4), with $H$ varying between 95 and $131 \mathrm{~mm}$. Here again, the influence of $H$ on water content profiles follows the same trend than that of $\mathrm{Hu}$ : for 95 and $113 \mathrm{~mm}$ the profiles are similar, while for $131 \mathrm{~mm}$ the water content is lower after the first third of the dryer. These trends in water content profiles result partly because of the higher contact area between sludge and the heated parts of the dryer for higher values of solids hold-up. However, a higher hold-up also implies a higher residence time of sludge: this parameter is thus also to be studied.

\section{Influence of hold-up on final water content}

The geometric residence time of dry solids $\tau_{d s}$ in the dryer can be calculated according to Eq. (8), where $Q_{d s}$ is the dry solids flow rate in the dryer.

$$
\tau_{d s}=H u / Q_{d s}
$$

The relation between $\tau_{d s}$ and sludge dry solids content at the outlet of the dryer is illustrated in Figure 11 for both geometric residence times obtained through experimental data and using Eq. 
(7). The final water content is the mean water content observed in sludge at the outlet of the dryer during the duration of the steady state. Experimentally, we observe a linear evolution between residence time and final water content until around $3 \mathrm{~h}$ of residence time, for which sludge is almost fully dried. Longer residence times are thus not required in our operating conditions. This highlights the importance of controlling sludge residence time in order to reach a desired water content at the outlet of paddle dryers, which will be determining for the development of economically feasible industrial energetic valorization for sludge [18].

In spite of the scattering between experimental and calculated residence time values due to the error on the correlation for $H u$ estimation (see Figure 7), the trends for experimental and calculated residence times are the same in Figure 11. The scattering in final water contents for similar residence times could be explained by the fact that different hold-ups can lead to the same residence time depending on the inlet sludge flow rate. Hence, for the same residence time, a higher loading of the dryer would result in higher final water content because of the increase in contact area between sludge and dryer walls. An accurate description of sludge drying in such reactors would require taking into account both the effect of residence time and of the contact area through which heat transfer would happen.

\section{Conclusion}

An empirical correlation relating sludge dry solids hold-up to paddle dryer operating parameters was determined thanks to a design of experiments. In a first step, a fractional factorial design was implemented, allowing studying the influence of 4 parameters with 8 experiments: sludge flow rate and stirring speed showed no influence on the hold-up, while installation slope, overflow 
height and their interaction were the most influential parameters. This design was then completed to a circumscribed central composite design with only these two parameters, giving access to a quadratic relationship between parameters and hold-up, over a larger range of operating conditions. The error on hold-up estimation by using this correlation is less than $15 \%$ compared to experimental data, and the standard error on calculated hold-up is $13.7 \mathrm{~g}$. The study of sludge water content profiles along the dryer confirmed that the influence of overflow height and slope installation on hold-up was not linear in our range of operating conditions. The determining role of hold-up is evidenced by the almost linear relationship between final dry solid content in sludge and its geometric residence time. These results will be implemented in a model describing heat and mass transfer during drying via the penetration theory along with sludge flow via a markovian approach.

\section{Nomenclature}

$\alpha, \beta \quad$ Coefficients for factors influence in DoE results

(-)

$H u \quad$ Dry solids hold-up in the paddle dryer

$H \quad$ Overflow height

$N \quad$ Stirring speed

(rpm)

$Q \quad$ Raw sludge flow rate

$Q_{d s} \quad$ Dry solids flow rate 
$R_{a}^{2} \quad$ Adjusted regression coefficient

$\theta \quad$ Dryer slope

$\tau_{d s} \quad$ Dry solids geometric residence time
$(-)$

\section{References}

1. Fytili, D., Zabaniotou, A.: Utilization of sewage sludge in EU application of old and new methods--A review. Renewable and Sustainable Energy Reviews. 12, 116-140 (2008).

2. Manara, P., Zabaniotou, A.: Towards sewage sludge based biofuels via thermochemical conversion - A review. Renewable and Sustainable Energy Reviews. 16, 2566-2582 (2012).

3. Kudra, T.: Sticky Region in Drying: Definition and Identification. Drying Technology. 21, 1457-1469 (2003).

4. Chen, G., Lock Yue, P., Mujumdar, A.S.: Sludge Dewatering and Drying. Drying Technology. 20, 883-916 (2002).

5. Arlabosse, P., Chavez, S., Lecomte, D.: Method for Thermal Design of Paddle Dryers: Application to Municipal Sewage Sludge. Drying Technology. 22, 2375-2393 (2004).

6. Arlabosse, P., Ferrasse, J.-H., Lecomte, D., Crine, M., Dumont, Y., Léonard, A.: Efficient Sludge Thermal Processing: From Drying to Thermal Valorization. In: Tsotsas, E. et Mujumdar, A.S. (éd.) Modern Drying Technology. p. 295-329. Wiley-VCH Verlag GmbH \& Co. KGaA (2011).

7. Rulkens, W.: Sewage Sludge as a Biomass Resource for the Production of Energy: Overview and Assessment of the Various Options $\uparrow$. Energy Fuels. 22, 9-15 (2008).

8. Tazaki, M., Tsuno, H., Takaoka, M., Shimizu, K.: Modeling of Sludge Behavior in a Steam Dryer. Drying Technology. 29, 1748-1757 (2011).

9. Charlou, C., Sauceau, M., Arlabosse, P.: Characterisation of Residence Time Distribution in a Continuous Paddle Dryer. J. Residuals Sci. Technol. 10, 117-125 (2013).

10. Milhé, M., Charlou, C., Sauceau, M., Arlabosse, P.: Modeling of sewage sludge flow in a continuous paddle dryer. Drying Technology (accepted for publication).

11. Box, G.E.P., Hunter, J.S., Hunter, W.G.: Statistics for experimenters: design, innovation, and discovery. Wiley-Interscience (2005).

12. Box, G.E.P., Hunter, J.S.: Multi-Factor Experimental Designs for Exploring Response Surfaces. The Annals of Mathematical Statistics. 28, 195-241 (1957).

13. Montgomery, D., C.: Design and Analysis of Experiments. Wiley.

14. Ferrasse, J., H., A., P...: Heat, momentum, and mass transfer measurements in indirect agitated sludge dryer. Drying Technology. 20, 749-769 (2002).

15. Charlou, C., Milhé, M., Sauceau, M., Arlabosse, P.: A new methodology for measurement of sludge residence time distribution in a paddle dryer using X-ray fluorescence analysis. Water Research (accepted for publication). 
16. Liu, X.Y., Specht, E.: Mean residence time and hold-up of solids in rotary kilns. Chemical Engineering Science. 61, 5176-5181 (2006).

17. Kramers, H., Croockewit, P.: The passage of granular solids through inclined rotary kilns. Chemical Engineering Science. 1, 259-265 (1952).

18. Ferrasse, J.-H., Seyssiecq, I., Roche, N.: Thermal Gasification: A Feasible Solution for Sewage Sludge Valorisation? Chem. Eng. Technol. 26, 941-945 (2003). 